ARTÍCULO ORIGINAL

\title{
Distribución anatómica de Rhipicephalus sanguineus sensu stricto (Acari: Ixodidae) sobre caninos domésticos
}

\author{
Orcellet $\mathrm{V}^{1^{*}}$, Nava $\mathrm{S}^{2}$ \\ ${ }^{1}$ Facultad de Ciencias Veterinarias, Universidad Nacional del Litoral (FCV-UNL), Argentina. \\ ${ }^{2}$ Estación Experimental Agropecuaria Rafaela, Instituto Nacional de Tecnología Agropecuaria, \\ Argentina. \\ * Correspondencia: Viviana Orcellet, FCV-UNL, RP Kreder 2805, Esperanza (CP B7000GHG), Argentina. E- \\ mail: orcellet@fcv.unl.edu.ar
}

Recibido: 18 Marzo 2020. Aceptado: 11 Junio 2020. Disponible en línea: 23 Junio 2020

Editor: P. Beldomenico

RESUMEN. En este trabajo se realizó el estudio de la distribución anatómica de los diferentes estadios de Rhipicephalus sanguineus sensu stricto sobre sus hospedadores principales, los caninos. El mismo se basó en muestreos mensuales durante tres años en la ciudad de Esperanza, Santa Fe, Argentina. Para determinar la distribución de los diferentes estadios parasitarios se dividió el cuerpo en regiones y se colectaron todos los especímenes en cada una de ellas. Las larvas tuvieron como preferencia las zonas de cabeza seguido de los espacios interdigitales, orejas y abdomen; las ninfas tuvieron preferencia por las orejas y abdomen, y otras zonas de preferencia fueron cabeza y espacios interdigitales; los adultos (machos y hembras) prefirieron localizarse en las orejas, cuello y lomo.

SUMMARY. Anatomical distribution of Rhipicephalus sanguineus sensu stricto (Acari: Ixodidae) on dogs. The anatomical distribution of the different stages of Rhipicephalus sanguineus sensu stricto on canines was analyzed. Data was collected from dogs on monthly sampling during three years in the city of Esperanza, Santa Fe, Argentina. To determine the distribution of the different parasitic stages, the body surface was divided into regions and all specimens were counted in each of them. Larvae had preference for the head followed by the interdigital spaces, ears and abdomen, nymphs were mainly found on ears and abdomen, and other areas of preference were head and interdigital spaces; the adults (males and females) preferred the ears, neck and back.

Palabras clave: Rhipicephalus sanguineus sensu stricto, distribución anatómica, caninos

Keywords: Rhipicephalus sanguineus sensu stricto, anatomical distribution, dogs

\section{Introducción}

Los ectoparásitos para poder alimentarse sobre su hospedador seleccionan sus lugares de ubicación en un sitio corporal para fijarse, el que puede constituir su hábitat final (Balashov, 1999; Krasnov et al., 2006). Los ectoparásitos muestran una predilección para colonizar determinadas áreas corporales de sus hospedadores para fijarse y alimentarse. El patrón de distribución resultante es la consecuencia de una interacción entre los parásitos y sus hospedadores, la coexistencia entre parásitos y la influencia de diferentes factores (Marshall, 1981; Nilsson, 1981; Reiczigel y Rózsa, 1998). Entre los factores específicos al parásito, se encuentran sus requerimientos para alimentarse y completar la fase parasitaria, competencia por los sitios de fijación, producción de feromonas que regulan la agregación, fijación, y procesos denso-dependientes como cambios en la distribución de acuerdo a diferentes densidades de individuos (Nelson et al., 1975; Rechav et al., 1976; Andrews y Petney 1981; Norval et al., 1989; Hamilton, 1992; Ogden et al., 2002; Soneshine, 2004; Wertheim et al., 2005; Braz Louly, 2008). Dentro de los factores propios del hospedador que pueden regular la distribución anatómica de sus ectoparásitos, se encuentran la respuesta activa al parasitismo por acicalamiento y la resistencia inmunológica adquirida, producción de feromonas con efectos tanto atrayentes como repelentes, variación de la temperatura corporal, sexo, edad, estatus reproductivo, estructura del pelaje y del tegumento, localización y densidad de las glándulas sebáceas (Nava et al., 2009).

El complejo Rhipicephalus sanguineus sensu lato, contiene un grupo de especies de garrapatas distribuida en ambientes urbanos y periurbanos. Las garrapatas del complejo $R$. sanguineus s.l, pueden ser vectores de Ehrlichia canis, Babesia canis vogeli, Babesia gibsoni, 
Rangelia vitalii (Babesia vitalii), Hepatozoon canis, Rickettsia rickettsii, Rickettsia conorii, Rickettsia massiliae, Anaplasma platys, Bartonella spp. y del filaroideo Dipetalonema reconditum (Otranto et al., 2009; Bowman, 2011; Labruna et al., 2011; Eiras et al., 2014). En el centro de la provincia de Santa Fe, la especie del complejo $R$. sanguineus que prevalece es Rhipicephalus sanguineus sensu stricto (Nava et al., 2012, 2018).

La importancia del conocimiento de los sitios de fijación de las garrapatas radica en el diseño de métodos de control efectivos con aplicación de acaricidas solo en los sitios predeterminados, minimizando el tiempo de muestreo al limitar la observación a las áreas donde se concentran los diferentes estadios de las garrapatas permitiendo una evaluación más precisa del número de éstas sobre un hospedador, facilitando así el desarrollo de estudios de la dinámica poblacional (Nava et al., 2009).

El objetivo de este trabajo fue analizar la distribución anatómica de los diferentes estadios de $R$. sanguineus s.s. (larvas, ninfas, adultos) sobre caninos (Canis lupus familiaris) para establecer si existen diferencias en la preferencia y en la variación de dicha distribución.

\section{Materiales y Métodos}

En tres lugares del Barrio La Orilla, de la ciudad de Esperanza, Santa Fe, Argentina, desde diciembre 2013 hasta diciembre 2016, se realizaron muestreos mensuales, un total de 37 , explorando la totalidad de las áreas del cuerpo de los caninos (entre 20 y 25 por mes) que no estaban circunscriptos al ámbito domiciliario, sino que deambulaban por el barrio, colectando cada uno de los diferentes estadios de $R$. sanguineus s.s., para verificar la distribución anatómica de las fases del ciclo. Todas las garrapatas colectadas se colocaron en recipientes adecuados con el agregado de alcohol 96으, para su posterior identificación, en el Laboratorio de Diagnóstico Parasitológico de la Facultad de Ciencias Veterinarias, utilizando lupas estereoscópicas. Las garrapatas se determinaron como pertenecientes a $R$. sanguineus s.s. siguiendo a Nava et al., 2018.

Para determinar la distribución anatómica de las garrapatas en los caninos, se tomó como base la clasificación utilizada por (Nava et al., 2009), dividiendo las áreas corporales de la siguiente manera: 1) Cabeza [CA]; 2) Orejas [OR]; 3) Cuello [CU]; 4) Pecho [PE]; 5) Lomo [LO]; 6) Abdomen, considerándose desde las axilas hasta la región inguinal $[A B]$; 7) Axila $[A X]$; 8) Miembro anterior [MA]; 9) Miembro posterior [MP]; 10) Espacio Interdigital [EI].

En la realización de los muestreos, se utilizó una ficha de campo donde se marcaron las áreas corporales de los caninos para el estudio de los sitios de fijación de los diferentes estadios de $R$. sanguineus s.s. (Figura 1).

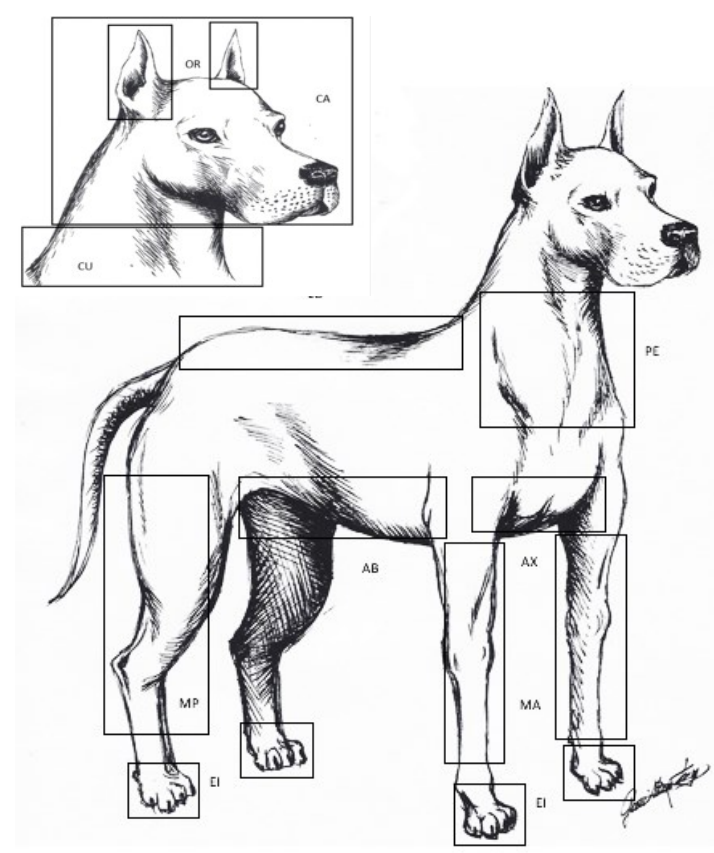

Figura 1. Esquema utilizado para el registro de garrapatas sobre los caninos. CA: cabeza; OR: oreja; CU: cuello; PE: pecho; LO: Lomo; $\mathrm{AB}$ : Abdomen; $\mathrm{AX}$ : Axila; MA: miembro anterior; MP: Miembro posterior; El: espacio interdigital. (Ilustración de Guillermo Jara).

En el estudio estadístico se aplicó la prueba de ShapiroWilk (Zar, 1999), para determinar si la distribución de los datos era normal. Debido a que los mismos no mostraron una distribución normal, la significancia de las diferencias entre las áreas corporales se testeó con métodos no paramétricos. Los valores obtenidos para cada área corporal se compararon estadísticamente con el test de Kruskal-Wallis seguido por la prueba de Dunn para comparaciones múltiples por pares (Zar, 1999).

\section{Resultados}

El total de observaciones realizadas sobre los caninos de los tres sitios muestreados fue de 762, de los cuales 523 presentaron al menos una garrapata. Sobre un total de 4681 especímenes colectados, 2187 fueron machos (46,5\%), 1522 hembras (32,5\%), 904 ninfas $(19,5 \%)$ y 68 larvas $(1,5 \%)$ de $R$. sanguineus s.s.

Se obtuvieron ejemplares de los 10 sitios corporales clasificados para el muestreo, CA: cabeza; OR: oreja; $\mathrm{CU}$ : cuello; PE: pecho; LO: Lomo; $\mathrm{AB}$ : Abdomen; $\mathrm{AX}$ : Axila; MA: miembro anterior; MP: Miembro posterior; El: espacio interdigital, los que se analizan cuantitativamente de acuerdo a cada estadio parasitario colectado.

\section{Distribución corporal de machos de R. sanguineus s.s.}

Se colectaron un total de 2187 machos de $R$. sanguineus s.s., en 408 observaciones realizadas sobre el total de caninos muestreados. Los mismos fueron colectados de todas las áreas corporales motivo de estudio. Cuando se consideraron para el análisis todas 
las observaciones (408) con el estadio de machos (2187), el análisis de Kruskal-Wallis indicó diferencias significativas entre las áreas $(P<0,01)$. El lugar preferido de localización fue $\mathrm{OR}$, y junto con las áreas CA, CU y LO constituyeron el grupo de áreas corporales, obtenidos con el procedimiento de Dunn, donde se concentra la infestación. La menor infestación estaría dada en las áreas AX, MA, MP y El (Tabla 1 ).

Tabla 1. Distribución corporal de los machos de $R$. sanguineus s.s. Cabeza [CA]; 2) Orejas [OR]; 3) Cuello [CU]; 4) Pecho [PE]; 5) Lomo [LO]; 6) Abdomen, considerándose desde las axilas hasta la región inguinal $[A B]$; 7) Axila [AX]; 8) Miembro anterior [MA]; 9) Miembro posterior [MP]; 10) Espacio Interdigital [EI].

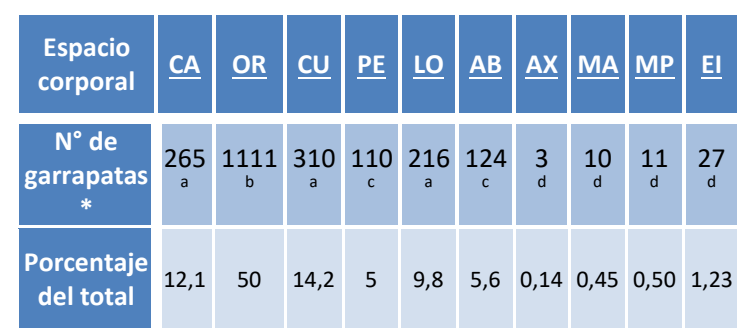

*Kruskal-Wallis con la prueba de Dunn para comparaciones múltiples por pares. Números seguidos por diferentes letras son estadísticamente diferentes $(P<0,01)$.

\section{Distribución corporal de hembras de R. sanguineus s.s.}

Para el caso de las hembras de $R$. sanguineus s.s., se encontraron en todas las áreas corporales. El total de observaciones recolectadas de los caninos muestreados durante el periodo de estudio fueron 762 de los cuales 379 presentaron al menos una hembra, colectándose un total de 1522 especímenes. Las mismas fueron colectadas de todas las áreas corporales motivo de estudio. Cuando se consideran los datos de todos los caninos parasitados con el estadio de hembras, el análisis de Kruskal-Wallis indicó diferencias significativas entre las áreas $(P<0,01)$. OR, CA, CU y LO constituyeron el grupo de áreas corporales donde se concentró la infestación. La menor infestación se observó en AX (Tabla 2).

Tabla 2. Distribución corporal de las hembras de $R$. sanguineus s.s. Cabeza [CA]; 2) Orejas [OR]; 3) Cuello [CU]; 4) Pecho [PE]; 5) Lomo [LO]; 6) Abdomen, considerándose desde las axilas hasta la región inguinal $[A B]$; 7) Axila $[A X]$; 8) Miembro anterior [MA]; 9) Miembro posterior [MP]; 10) Espacio Interdigital [EI].

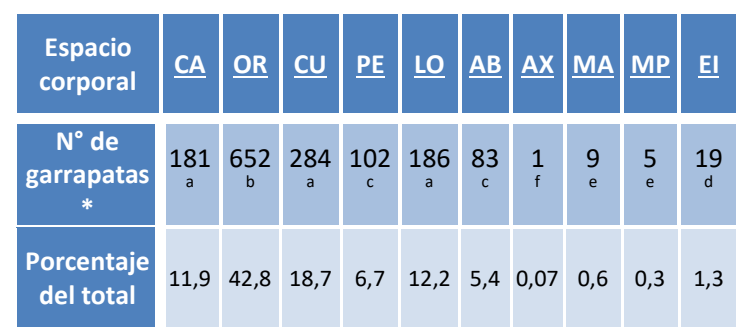

*Kruskal-Wallis con la prueba de Dunn para comparaciones múltiples por pares. Números seguidos por diferentes letras son estadísticamente diferentes $(P<0,01)$.
Tanto para machos como para hembras de $R$. sanguineus s.s., el lugar de preferencia para su localización, y de acuerdo a lo que indica la estadística son las orejas.

\section{Distribución corporal de ninfas de R. sanguineus s.s.}

Se encontraron ninfas en todos los lugares con excepción de los miembros anteriores (MA). De las 523 observaciones de caninos que estaban parasitados con garrapatas, 164 presentaban la fase de ninfa. Se colectaron en total 904 de ellas durante el periodo muestreado. Los sitios preferidos para la fijación son $O R, A B$ y $C A$ (Tabla 3$)$.

Tabla 3. Distribución corporal de las ninfas de $R$. sanguineus s.s. Cabeza [CA]; 2) Orejas [OR]; 3) Cuello [CU]; 4) Pecho [PE]; 5) Lomo [LO]; 6) Abdomen, considerándose desde las axilas hasta la región inguinal $[A B]$; 7) Axila $[A X]$; 8) Miembro anterior [MA]; 9) Miembro posterior [MP]; 10) Espacio Interdigital [EI].

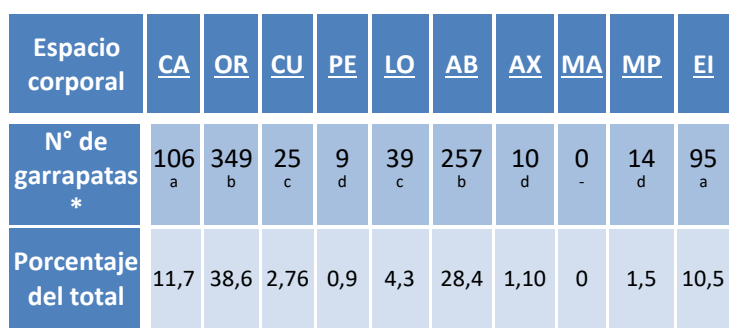

*Kruskal-Wallis con la prueba de Dunn para comparaciones múltiples por pares. Números seguidos por diferentes letras son estadísticamente diferentes $(P<0,01)$.

\section{Distribución corporal de larvas de R. sanguineus s.s.}

Para el caso de las larvas, de los lugares muestreados, no se hallaron ejemplares en AX, MA y MP. De las 523 observaciones que presentaban al menos una forma parasitaria durante el periodo de muestreo, 20 presentaban larvas, colectándose un total de 68 de ellas. El análisis de Kruskal-Wallis indica diferencias significativas entre las áreas $(P<0,01)$. El lugar preferido de localización fue CA, seguido de El, OR y AB (Tabla 4).

Tabla 4. Distribución corporal de las larvas de $R$. sanguineus s.s. Cabeza [CA]; 2) Orejas [OR]; 3) Cuello [CU]; 4) Pecho [PE]; 5) Lomo [LO]; 6) Abdomen, considerándose desde las axilas hasta la región inguinal [AB]; 7) Axila [AX]; 8) Miembro anterior [MA]; 9) Miembro posterior [MP]; 10) Espacio Interdigital [EI].

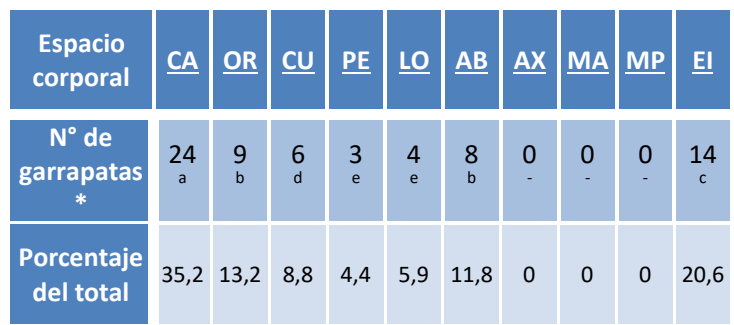

*Kruskal-Wallis con la prueba de Dunn para comparaciones múltiples por pares. Números seguidos por diferentes letras son estadísticamente diferentes $(P<0,01)$. 


\section{Discusión}

Se tomaron especímenes de $R$. sanguineus s.s. en todas las regiones corporales estudiadas. La mayor cantidad de garrapatas se colectaron en $\mathrm{OR}$, mientras que el menor número se encontraron en axilas (AX) y miembros (MA, MP). Esto difiere de los hallazgos de (Moissant et al., 1999), donde la mayor localización se dio en cuello.

Entre las diez regiones corporales el orden de preferencia fue el siguiente: OR, CA, CU y LO, con las mayores cantidades de especímenes de adultos $(P<0,01)$. La menor preferencia para todos los estadios, fueron $\mathrm{AX}, \mathrm{MA}$ y $\mathrm{MP}(\mathrm{P}<0,01)$, hecho que coincide con los hallazgos de (Moissant et al., 1999).

Si bien se colectó un mayor número de machos, se encontraron machos y hembras en todas las regiones corporales. Estos resultados parecen estar relacionados con la actividad sexual de los adultos en cuanto a la producción de feromonas sexuales por parte de la hembra durante el proceso de ingurgitación, con la finalidad de atraer los machos y realizar la cópula (Hamilton, 1992).

Los estados inmaduros se colectaron en menor cantidad, pero también mostraron preferencia por las regiones de abdomen y espacios interdigitales (AB, EI). Las ventajas de este comportamiento son la fijación a una superficie tegumentaria delgada con alta vascularización y que en éstas áreas la defensa mecánica del hospedador por acicalamiento tiene un menor efecto que en otras áreas corporales (Nava et al., 2009).

La distribución anatómica de las diferentes fases parasitarias de $R$. sanguineus s.s., abarcó la totalidad de las áreas corporales de los caninos que participaron en el estudio, con excepción de la cola.

\section{Bibliografía}

Andrews RH, Petney T N. 1981. Competition for sites of attachment to host in three parapatric species of reptile tick. Oecologia 51: 227-232.

Balashov YS. 1999. Evolution of haematophagy in insects and ticks. Entomol. Rev. 79: 943-954.

Bowman DD. 2011. Introduction to the Alphaproteobacteria: Wolbachia and Bartonella, Rickettsia, Brucella, Ehrlichia, and Anaplasma. Top. Comp. Anim. Med. 26: 173-177.

Braz Louly CC, Nóbrega Silveira D, Fernandes Soares S, Ferri PH, Campos De Melo AC, Ferreira Borges LM. 2008. More about the role of 2,6-dichlorophenol in tick courtship: identification and olfactometer bioassay in
Amblyomma cajennense and Rhipicephalus sanguineus. Mem. Inst. Oswaldo Cruz 103: 60-65.

Eiras DF, Craviotto MB, Baneth G, Moré G. 2014. First report of Rangelia vitalii infection (canine rangeliosis) in Argentina. Parasitol. Int. 63: 729-34.

Hamilton JGC. 1992. The role of pheromones in tick biology. Parasitol. Today 8: 130-133.

Krasnov BR, Poulin R, Morand S. 2006. Patterns of macroparasite diversity in small mammals. En: Morand S., Krasnov B. R., Poulin, R. (eds). Micromammals \& Macroparasites. From evolutionary ecology to management. Springer-Verlag, Tokyo. Pp. 197-231.

Labruna MB, Mattar S, Nava S, Bermudez S, Venzal JM, Dolz G. 2011. Rickettsioses in Latin America, Caribbean, Spain and Portugal. Rev. MVZ Córdoba. 16: 2435-2457.

Marshall AG. 1981. The ecology of ectoparasitic insects. New York Academic Press, New York, 459 pp.

Moissant E, Vicente M, García Y, Armas S. 1999. Estudio bioecológico de la garrapata del perro, Rhipicephalus sanguineus (Acari: Ixodidae), en un criadero en El Limón (Edo. Aragua), Venezuela. Rev. Fac. Cs. Vet. UCV 40: 119-125.

Nava S, Mangold AJ, Guglielmone AA. 2009. La distribución anatómica de Amblyomma neumanni y Amblyomma parvum (Acari: Ixodidae) sobre sus hospedadores principales. FAVE Sección Ciencias Veterinarias 8: 11-21. https://doi.org/10.14409/favecv.v8i2.1483

Nava S, Mastropaolo M, Venzal J M, Mangold AJ, Guglielmone AA. 2012. Mitochondrial DNA analysis of Rhipicephalus sanguineus sensu lato (Acari: Ixodidae) in the Southern Cone of South America. Vet. Parasitol. 21: 547-555.

Nava S, Beati L, Venzal JM, Labruna MB, Szabo MPJ, Petney TN, Saracho Bottero MN, Tarragona EL, Dantas Torres F, Santos Silva MM, Mangold AJ, Guglielmone AA, Estrada-Peña A. 2018. Rhipicephalus sanguineus (Latreille, 1806): Neotype designation, morphological re-description of all parasitic stages and molecular characterization. Ticks Tick-borne Dis. 9: 1573-1585.

Nelson WA, Keirans JE, Bell JF, Clifford CF. 1975. Hostectoparasite relationship. J. Med. Entomol. 13: 143-166.

Nilsson A. 1981. Spatial differentiation of ectoparasites on small mammals. Holarct. Ecol. 4: 184-190.

Norval RAI, Andrew HR, Yunker CE. 1989. Pheromonemediation of host-selection in bont ticks Amblyomma hebraeum (Koch). Science 243: 364-365.

Ogden NH, Casey ANJ, French NP, Adams JDW, Woldehiwet Z. 2002. Field evidence for density- 
dependent facilitation amongst Ixodes ricinus ticks feeding on sheep. Parasitology 124: 117-125.

Otranto D, Dantas Torres F, Breitschwerdt EB. 2009. Managing canine vector-borne diseases of zoonotic concern: part one. Trend. Parasitol. 25: 157-163

Rechav Y, Whitehead GB, Knight MM. 1976. Aggregation response of nymphs to pheromone(s) produced by males of the tick Amblyomma hebraeum (Koch). Nature 259: 563-564.

Reiczigel J, Rózsa L. 1998. Host-mediated site segregation of ectoparasites: an individual-based simulation study. J. Parasitol. 84: 491-498.

Sonenshine DE. 2004. Pheromones and other semiochemicals of ticks and their use in tick control. Parasitology 129: 405-425.

Wertheim B, Van Baalen E J A, Dicke LE. 2005. Pheromone-mediated aggregation in nonsocial arthropods: an evolutionary ecological perspective. Ann. Rev. Entomol. 50: 321-346.

Zar JH. 1999. Biostatistical analysis. $4^{\text {ta }}$ ed., PrenticeHall, New Jersey. 663 pp. 